\title{
Antiviral Targeting of NF-kB: A Potential Therapy for HIV and Herpesvirus Associated Lymphomas
}

William J. Harrington, Jr.

NF-kB refers to a group of proteins involved in inflammatory, immune, and pro- and anti-apoptotic cellular responses. NF-kB mediated transactivation occurs through assembly of monomeric subunits at $\mathrm{kB}$ binding sites. In the classical pathway inhibitor of Kappa B ( $\mathrm{IkB}$ ) segregates NF-kB in the cytoplasm preventing entry to the nucleus. In response to a variety of stimuli, including cytokines and viral infection, IkB is phosphorylated by the IkB kinase complex (IKK) and degraded by the ubiquitin proteosome pathway, allowing for nuclear localization and DNA binding. The induction of NF-kB by the EBV oncoprotein LMP-1 has been well established. LMP-1 signaling activates NF-kB through a mechanism that mimics CD40 and protects B cells from apoptosis. Although CD40 and lymphotoxin beta receptor (LTßR) both activate NF-kB, recent studies have demonstrated that the mechanism of action in each differs.

Constitutive activation of NF-kB is an important anti apoptotic mechanism in poor prognosis B cell lymphomas. Several groups have recently demonstrated that inhibition of NF-kB in gamma herpes virus associated lymphomas results in induction of apoptosis. In addition, blockade of NF-kB has been demonstrated to disrupt gamma herpes viral latency.

We previously reported on the activity of antiviral agents in certain herpesvirus associated lymphomas. Most of our work has centered on the activity of the antiviral thymidine analogue, AZT, alone or in combination with interferon alpha (IFN- $\alpha$ ).

We have studied how the antiviral agents AZT and IFN- $\alpha$ induce apoptosis in herpesvirus associated lymphomas. EBV+ BL's undergo apoptosis in the presence of AZT whereas viral negative lymphoma lines are unaffected. AZT is phosphorylated to AZT-MP in EBV+ BL's. This compound (AZT-MP) appears to be a potent inhibitor of NF-6B.

Recently we have demonstrated that AZT (AZT-MP) blocks NF-6B nuclear activity in PEL. When coupled with IFN-” induced TRAIL this produces striking apoptosis. This also may explain why IFN-” alone only weakly induces apoptosis in PEL. We have found that TRAIL (or IFN- $\alpha$ ) actually activates NF-kB, thereby blunting its pro-apoptotic effect. In contrast, AZT (AZT-MP) blocks NF-kB, thus potentiating the pro-apoptotic effect of IFN- $\alpha$. This activity appears to result from AZT mediated inhibition of IKK which results in sequestration of NF$\mathrm{kB}$ in the cytoplasm. We have recently found that AZT treatment of EBV+ BL cells inhibits NF-kB activity within 2 to 4 hours followed by the induction of the lytic cycle of EBV (assessed by an EBV quantitative RT-PCR array and immunoblots). Both viral kinases, EBV- thymidine kinase (TK) and BGLF-4, were induced by AZT. In AZT treated EBV+ BL but not in EBV- BL there was a marked increase in phosphorylation of the antiviral nucleoside and a pronounced apoptotic effect. AZT, an antiviral, paradoxically induces the viral lytic cycle and subsequent apoptosis in EBV+ BL and, is a novel targeted therapeutic agent for these aggressive tumors. Recently, we have devised a simple strategy to enhance the anti-lymphoma activity of AZT in herpesvirus lymphomas. Hydroxyurea potentiates the phosphorylation of nucleosides through inhibition of ribonucleotide reductase. The in vitro phosphorylation and potentiation of the apoptotic effect of AZT on EBV+ BL is markedly enhanced in the presence of hydroxyurea. We have used this successfully to treat patients with AIDS related EBV+ lymphoproliferative disease. We envision that this simple, inexpensive combination may prove to be very active for $\mathrm{EBV}+$ (endemic) BL.

Our clinical and laboratory data demonstrate that herpesvirus-associated lymphomas in immunosuppressed individuals are uniquely sensitive to AZT or AZT and IFN- $\alpha$. Effective therapy for these deadly tumors has been lacking. In transplant recipients, hereditary immunodeficiency and AIDS, the use of antivirals rather than 
immunosuppressive cytotoxic chemotherapy is likely to improve the outcome for these patients. Further clinical and basic studies should demonstrate the role of these agents in diseases such as post transplant and endemic Burkitt’s lymphomas.

\section{Epidemiology of Virus-Associated Cancers in Brazil Rocío Hassan}

Epidemiological evidences point to an increasing causative role of viruses in the multifactorial multistep etiology of human malignancies. The interactions of viruses with certain environmental and genetic factors may account for the geographic variability of incidences and clinic-biological characteristics of particular virus-associated cancers. Estimates about the preventable risks of cancer in the U.S. showed that approximately $10 \%$ of human cancers were causatively associated with viruses. In developing countries, interaction with other etiologic and risk factors besides the size of the "at risk" populations ( $80 \%$ of human population), act synergically to increase the magnitude of this public health problem. In most of the cases, an accurate characterization of the problem is hampered by the lack of interdisciplinary studies in those areas. In Brazil, the main associations are HPV and cervical cancer, HTLVI/II and T cell leukemia and EBV -associated neoplasms, all of them transversally influenced by the HIV epidemics. Due to the continental dimensions of the country, investigations on prevalence, viral infection patterns and association with other environmental, genetic and susceptibility factors are needed, on a regional basis, for identifying risk areas and virus-host specific interactions involved in viral associated carcinogenesis, aiming to the design of prevention and therapeutic strategies.

We studied a group of 62 children with Burkitt's lymphoma (BL) at the National Institute of Cancer, (INCa), Rio de Janeiro. Association with Epstein-Barr Virus (EBV) was found in 60\% of the cases (86\% of type 1), thus confirming an intermediate prevalence in the Southeast region of the country, compared to the Northeast region, where it can reach 87\%. The four children diagnosed with AIDS-BL were EBV-positive. Children with EBV+ $B L$ were younger than those with $\mathrm{EBV}-\mathrm{BL}(\mathrm{p}=0.002)$. Aiming to identify susceptibility factors, we studied the IL10 promoter polymorphisms SNP-1082(G/A) and STR IL10.G, as well as TNF $\alpha$ SNP-308(G/A). We found an excess of the highly expressing allele SNP-1082 (G) in EBV-negative patients (OR 2.82; CI 95\% 1.22-6.51; $\mathrm{p}=0.013$ ) pointing to a stimulatory, pro-oncogenic role of the IL-10 in this group. In 15 BL patients we investigated the expression of cellular genes by RT-PCR and found that cyclin D2 was expressed in $53 \%$ of the cases, associated to the presence of EBV ( $\mathrm{p}=0.041)$. BCL2 was expressed in 4 of 13 cases (31\%). Expression of activation induced cytidine deaminase (AID) gene, which is involved in physiological maturation and remodelling of immunoglobulin genes was quantified by Real-time PCR. We found a higher than expected expression of AID in the EBV-positive cases, not associated to the presence or degree of somatic hypermutation of IGH locus, which may be reflecting interactions of viral and cellular regulatory pathways. In sum, we presented results of current research in our laboratory which is helping us to understand the complex interactions between virus and host in the pathogenesis of Burkitt‘s lymphoma in this region.

\section{Epidemiology of AIDS-Related Malignancies in Bahia Eduardo M Netto, Estela Luz, Carlos Brites}

Brazil reported up to June 2004 more than 362,000 individuals with AIDS (and 160,000 deaths) most of all with full blow disease, with a cumulative incidence rate of $18.4 / 100,000$ habitants. The number of cases is rather 